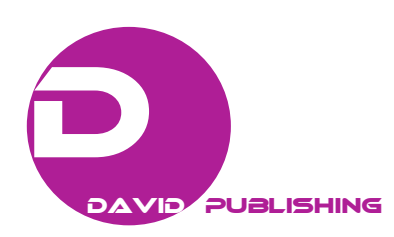

\title{
Architectural Teaching and Design Computing: A Didactic Experience in a Brazilian Architecture Course
}

\author{
Fernando Tadeu de Araujo Lima \\ Faculty of Architecture and Urbanism (FAU) and Post-Graduate Program of Built Environment (PROAC), Federal University of \\ Juiz de Fora, Juiz de Fora, Minas Gerais, 36037-000, Brazil
}

\begin{abstract}
In the last years, architectural practice has been confronted with a paradigm shift towards the application of digital methods in design activities. In this regard, it is a pedagogic challenge to provide a suitable computational background for architectural students, to improve their ability to apply algorithmic-parametric logic, as well as fabrication and prototyping resources to design problem solving. This challenge is even stronger when considering less favored social and technological contexts, such as in Brazil, for example. In this scenario, this article presents and discusses the procedures and the results from a didactic experience carried out in a design computing-oriented discipline, inserted in the curriculum of a Brazilian architecture course. Hence, this paper shares some design computing teaching experiences and presents some results on computational methods and creative approaches, with a view to contribute to a better understanding about the relations between logical thinking, mathematics and architectural design processes.
\end{abstract}

Key words: Architectural design teaching, design computing, didactic experience.

\section{Introduction}

Several studies have addressed teaching methodologies towards a more creative use of the computer in architectural tasks. In this regard, Achten [1] presents some teaching methods, and the works of Al-Ratrout and Zureikat [2] and Aguiar and Gonçalves [3] shows some recently implemented approaches. In a Brazilian context, although there are some initiatives in this direction, there is still a little space for implementing computational content in architecture courses curricula.

The present work is a result of a research that aims to contribute to a better understanding about how computational resources can be incorporated in the teaching and the practice of architecture in contemporary times, more specifically, in a Brazilian context. In this regard, it is a pedagogic challenge to provide a suitable conceptual and instrumental

Corresponding author: Fernando Tadeu de Araujo Lima, Ph.D.; research fields: parametric design, architectural design, and urban design. E-mail: fernando.lima@arquitetura.ufjf.br. background for architectural students, as well as to stimulate them to resort logical thinking and computational resources to design problem solving. This challenge is even stronger when considering less favored social and technological contexts, such as in Brazil, for example. In this scenario, this article aims to contribute towards a discussion about the importance of design computing in the knowledge and skills of architecture and urbanism students and, consequently, in the curricular content of undergraduate courses of architecture. Thus, this paper articulates a theoretical framework on computational resources supporting architectural design methods [4-8] and describes the procedures and presents the results from a didactic experience, carried out in a design computing-oriented discipline, inserted in the curriculum of a Brazilian architecture course. In this discipline, called digital modeling and prototyping, the students were introduced to theories, concepts, resources and techniques related to computational design implementation, in order to promote logical reasoning and abstraction approaches 
in architectural design tasks. These students were submitted to theoretical lessons, algorithmic-parametric modeling classes and were also introduced to rapid prototyping and digital manufacturing use, such as 3D printers and laser cutter machines. In this sense, the objective of this pedagogical approach was to broaden students' creative repertoire, as well as to stimulate their interest in research with a view that in the future they will be able to apply computational practices in their design methods.

Therefore, this paper aims to contribute with some reflections on teaching design computing methods and creative approaches for architectural purposes, with the view to describe and discuss about the developed exploration, which consisted of a didactic experiment carried out in the above-mentioned discipline, and lasted two academic semesters, with four classes. They were approximately 90 students, mostly in the third semester (2nd year) of the course. In the discipline, more specifically, the students: (1) were introduced to concepts, theories and resources related to computational logic in support of design tasks, in a seminar where they discussed and presented themes related to the computer-aided architectural design universe; (2) were taught to use algorithmic-parametric logic software, through a pedagogical approach that aimed at the abstraction and the modelling of iconic buildings, in order to provide a visual programming language modeling background; (3) elaborated a final project in which they should apply the design computing techniques learnt in a design task; and (4) were submitted to a survey about the contributions of the discipline, in which they answered on the advantages and disadvantages of applying computational resources in their design practices, and if they intended to use design computing techniques again. By demonstrating the real possibilities brought by advances in design computing techniques, such as parametric design software, as well as the use of rapid prototyping applied to architecture, this pedagogical approach aimed to boost up knowledge and skills of architecture students, even if they are in less favored social and technological contexts. As final remarks, this paper: (1) articulates the obstacles encountered and the results obtained with the students' final works; (2) presents the students' perceptions regarding the possibilities of using computational resources; and (3) lists further developments for this research and for the teaching approach itself.

\section{Theoretical and Conceptual Basis}

According to Menges [5], the tools architects adopt steer their design process. In this regard, computational approaches introduce an important change in design methods, as long as they allow (and at the same time demand) a different logic of thinking. On the other hand, Mitchell [4] states that the act of designing as a discipline emerges as a way of abstracting and evaluating different alternatives and scenarios, without necessarily perform physically each possible solution.

Terzidis [8], in turn, identifies the conventional design systems as obsolete, since they follow a logic of drawing and erasing, doing and undoing. In this context, the algorithmic-parametric modeling paradigm introduces a fundamental change in the design process logic, as long as it allows parts of a given project to be dynamically related. That is, the relationships are explicit, and components change in a coordinated way, in response to each parameter modification. In this sense, to code parametric algorithms means to link data, conditions and variables, and it implies thinking about and describing the relationships between each part. Thus, relating parametric and algorithmic resources, and their capability to manage data in a dynamic way, can support interactive and more efficient design approaches.

Still according to Terzidis [8], parametric modelling is programming, and therefore, using this 
logic requires an advanced understanding of geometry, computing and the structure of parametric systems themselves. In this regard, apart from controlling algorithmic and scripting issues, it is also important to master geometric knowledge. In the meantime, Woodbury [6] points that algorithms allow designers to deal with complexity in a level that is beyond regular human capacity. Tedeschi [7], in turn, defines algorithm as a procedure for addressing a problem in a finite number of steps using logical if-then-else operations. In this sense, this paradigm shift in architectural practice requires new pedagogical approaches to be proposed, in order to allow the academic context to follow the new possibilities of professional practice.

Architectural design has always had a strict relationship to the available techniques at times. Computational design tools with support for digital manipulation, in this sense, enables a wide range of experiences that relate not only to representation, but essentially to the design process itself. Therefore, teaching computational content, in an architectural perspective, refers directly to design methods and not only for representation tasks. The didactic experience presented in this paper, took this argument as a premise.

\section{The Didactic Experience}

The didactic experience developed can be structured through three stages, namely: (1) theoretical-conceptual introduction-stage in which the students were presented to key terms and concepts related to computational implementation in architecture and urbanism, through an internal seminar of the discipline where several themes were addressed, such as "parameterization", "algorithm", "optimization", "generative design", "design computing" among others; (2) abstraction logic and parametric algorithmic modeling - introduction to modeling logic in Rhinoceros + Grasshopper software, in which students were introduced to different commands and scripts, through the shape abstraction, modeling and 3D printing of different iconic buildings, in a clear intention to familiarize them with the theme of the discipline and to broaden their computational repertoire; and (3) final work of the discipline - elaboration of a kind of synthesis panel of the learning process of the discipline, which consisted in the proposition of two different products, obtained in two different semesters, namely: (a) the design of different shelters that should meet diverse climatic demands, according to different locations, in the first semester experience; and (b) the design, fabrication and assembly of a "catenary-3D voronoi" arch, in the second semester experience. In these final works, it was fundamental condition to use algorithmic-parametric modeling resources to develop the proposals. The students also had 3D printers and laser cutting machines available for work.

Hence, this didactic experience begins with introducing students to digital modeling and prototyping universe, which is developed through the presentation of different modeling commands and resources (supported by the abstraction and decomposition of paradigmatic buildings shape) and culminates with an attempt to implement algorithmic-parametric resources in design tasks developed by the students themselves.

The first stage of the discipline (essentially theoretical) proved to be important, because in a general way, students are easily attracted to propose unusual (non-Euclidean) shapes but have little knowledge about the concepts behind the geometrical and tectonic formulations adopted. In addition, the parametrization topics that were addressed allowed students to arouse interest in new possibilities for creative processes, with unexpected discoveries arising from the possibility of manipulating form-generating parameters.

The second stage, which contains a large part of the discipline's timetable, aimed to broaden students' propositional repertoire. It consisted on a series of 
classes in which iconic buildings had their shapes abstracted for the construction of algorithmic definitions for their modeling (e.g., Swiss Re Headquarters, Absolute Towers, Holocaust Memorial). The first tasks were oriented with the help of the teacher and the monitors of the discipline, aiming at the development of simple solutions from the examples shown in class, where each student made small variations based on the values and constraints of the codes. The classes covered a gradual teaching of the addressed tools and operations, seeking to promote an increasing acquisition of computational repertoire and a greater familiarity with mathematical concepts and the interface of the software, so that the students could, firstly, understand the processes described and the logic behind the commands and, later, reinterpret them and adapt them to several situations that could be presented. In this context, the dynamic of the classes was based on the introduction of different formal concepts, followed by practical exercises of modeling and prototyping different iconic buildings, as shown in Fig. 1.

The third stage, which consisted in the application of a final work for the discipline, was aimed at verifying how students would implement the concepts, techniques and resources presented during the semester in practical design situations.

Considering that this didactic experience lasted two academic semesters and that in each semester different final works were proposed, this third stage had two different approaches, namely: (1) in the first approach, the students (divided into groups of four components) were asked to design a shelter located in different biomes (e.g. tropical forest, tundra, polar region, desert, city, savannah, beach, among others). The proposed shelters should meet different climatic constraints and, at the same time, explore algorithmic-parametric logic in its development; in the second approach, the proposal was for the whole class to work on designing, modeling, manufacturing and assembling a final product: a 3D voronoi catenary arch. Thus, these two different approaches allowed to visualize different contexts of work, including design conceptualization, teamwork, digital modeling, prototyping fabrication and assembly of components.

In this sense, the final work of the discipline consisted of design tasks that could be accomplished (from conception to prototyping, with the guidance of the professor in the classroom) in the period of three classes (considering a class per week).

Thus, the purpose of performing a design exercise using algorithmic-parametric modeling was to promote a new design approach in students' reality, since they had not yet explored such resources in their training. By using a modeling logic, based on the definition and relation of parameters, the students

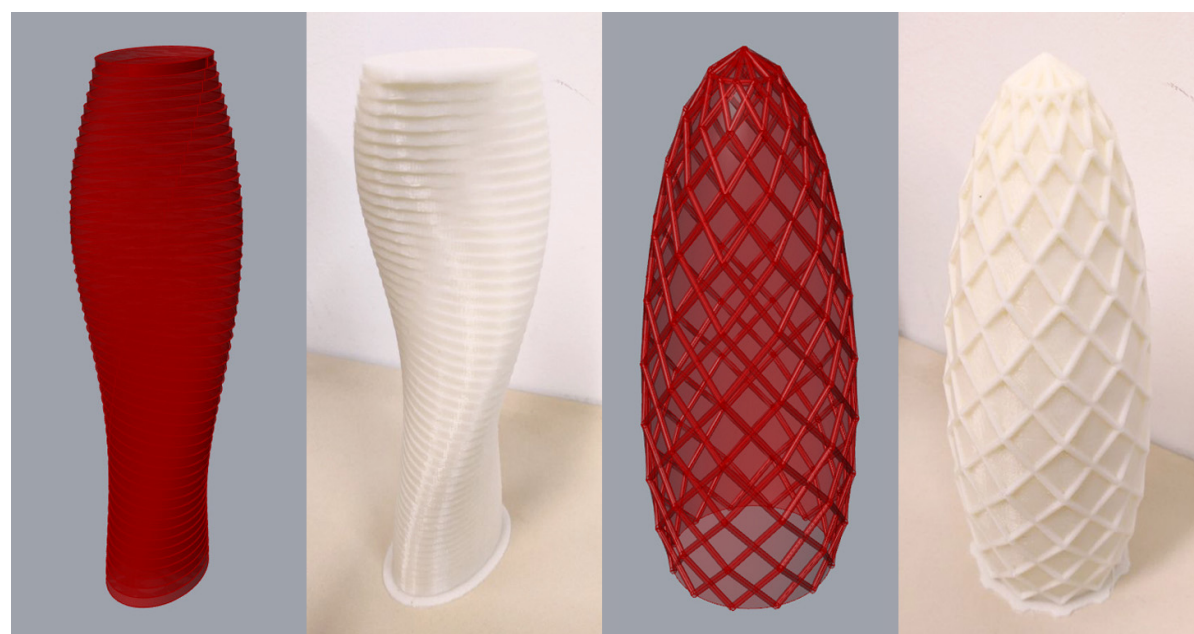

Fig. 1 Algorithmic-parametric models (and their 3D printed prototypes) developed by the students and inspired by different iconic projects. 
were faced with another possibility for architectural design tasks, which involves abstraction, logical reasoning and the parameterization of distinct and variable attributes.

At the end of the course, there was an evaluation of the discipline, carried out in a conversation with the students and through an online evaluation form, which they should fill anonymously.

\section{Results}

The results obtained in this didactic experience, which, as previously mentioned, lasted one year (two separate classes of one semester each), can be organized according to the following sequence: (1) the results from Approach 1 (first semester of 2017); (2) the results form Approach 2 (second semester of 2017); and (3) the overall students' evaluation of the discipline.

\subsection{Approach 1}

In the first semester of 2017 final works, the diversification of the shelters' biomes resulted in different and interesting solutions. All groups were able to identify attributes that could be parameterized, in order to obtain solutions that could fit the conditions of each specific site. Therefore, it was possible for the students to find great utility in developing projects through algorithmic-parametric logic.

In this context, we highlight the works in which the authors went beyond the content taught, and sought to use resources not presented in class, such as the use of add-ons as Ladybug (used for environmental comfort analysis) or Lunchbox (used to explore geometric shapes), for example. That is, it was possible to perceive that some groups have explored the use of scripts and geometric descriptions for mass and shape study, not only aiming at aesthetic aspects, but also looking at aspects such as thermal performance, constructability data analysis, and structure optimization. Among the procedures that the students used to parameterize attributes of different shelters and their respective solutions, we can highlight some of them: (1) the use of the Ladybug add-on to guide the optimization of openings positioning for a shelter in the polar region, seeking to find a configuration with greater use of radiation and heat; (2) the development of a script to quantify the required number of adobe bricks for building a shelter, considering the use of local materials and their location on the African savanna; (3) the use of Lunchbox add-on to evaluate which "geometric matrix" would result in a more appropriate aesthetic result for a shelter in the Mexican desert of Sonora; (4) the construction of a definition of a geometric shape and its structural diagrid, based on a shell, in a clear biomimetic reference for a shelter on a Brazilian beach; (5) the configuration of a set of containers adapted as shelter and stacked so as to make better use of the sun and the wind in an urban center. Some examples of the elaborated works are shown in Fig. 2.

\subsection{Approach 2}

The second semester of 2017 final work, aimed at verifying students' ability to work as a team and to use the discipline content for designing, modeling, manufacturing and assembling a final product. In this sense, the students proposed a catenary arch, subdivided into 3D voronoi cells, for production. Thus, the arch was designed, modeled and divided into 36 voronoi $3 \mathrm{D}$ pieces, in a way that each student was responsible for customizing and unrolling the faces of one piece for laser cutting. After all, each student assembled one piece, and in the final class of the semester, the prototyped pieces were put together in a $1 / 2$ scale model. This approach was interesting because it allowed students to participate in a large design process, from conception to assembly. In addition, it was important for each student to know that their piece was part of a whole and that each proposed customization would have implications on the outcome of the work. Fig. 3 illustrates the digital 


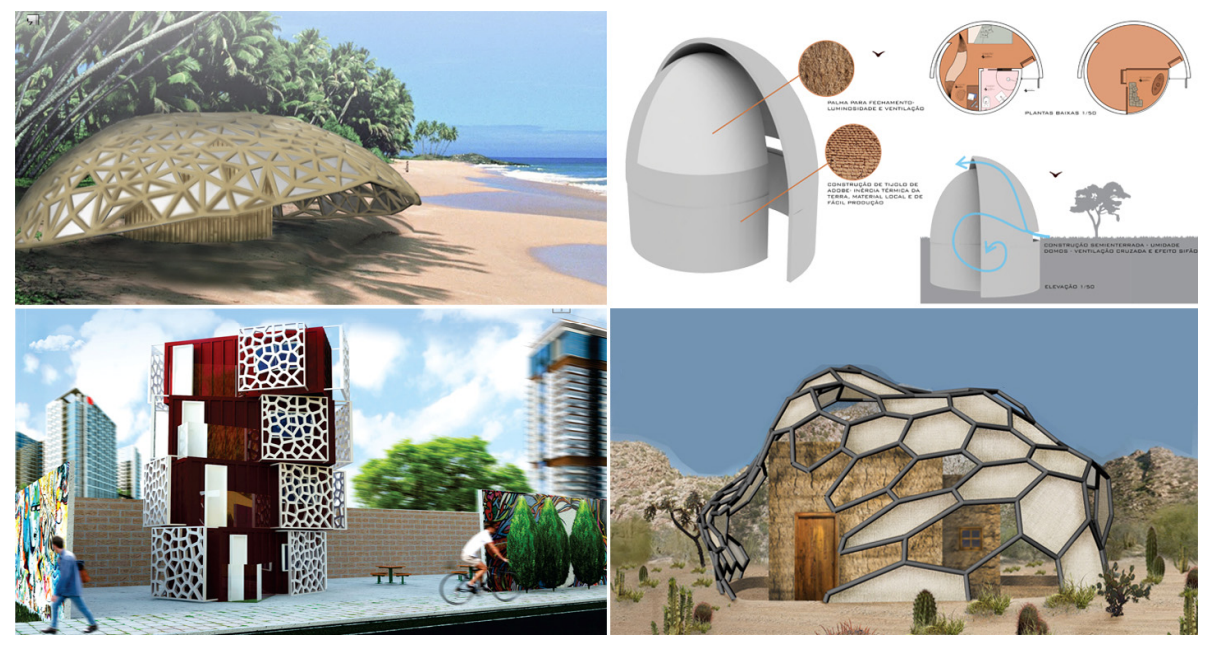

Fig. 2 Some examples of the elaborated shelters in the final work of the discipline.

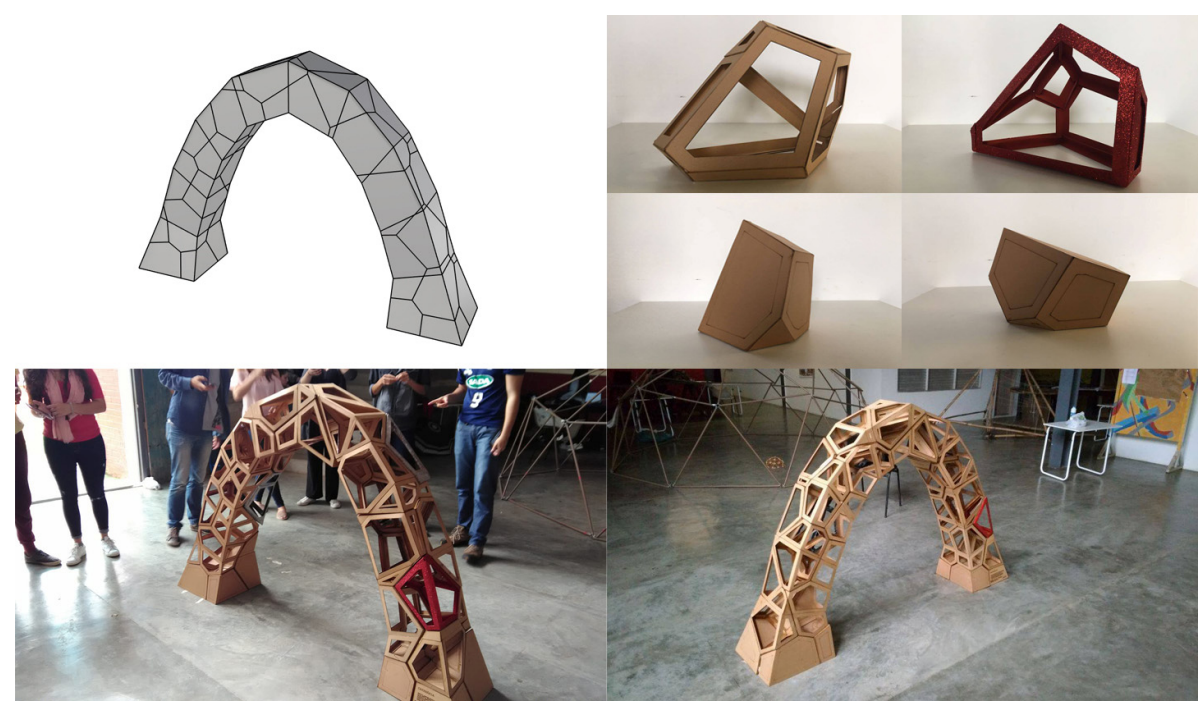

Fig. 3 The digital model, some separate parts, and the finished arch in a 1/2 scale.

model of the proposed arch, some separate parts, the assembly process and the finished arch.

\subsection{Students' Evaluation}

In order to evaluate the discipline's contributions to the students' development, and also to get a feedback from them, an online evaluation form was made available. In this form, the students were anonymously asked about: (1) the relevance of the discipline's content, regarding architecture and urbanism in contemporary times; (2) their degree of interest about the resources, the tools and the possibilities of design development presented in the discipline; (3) their level of difficulty in understanding and performing the proposed activities in the classroom; (4) the usefulness of the content taught for the development of architecture projects; (5) if they pretend to continue using the discipline's contents in the future; (6) how adequate was the teaching time, in the whole semester; and (7) which resources presented were of major importance.

The questionnaire was structured under the logic of SEQ (single ease question), in a 7-point rating scale to assess the students' opinions. Hence, it was possible to work mathematically with the answers obtained and to get some hints about their opinions. For an example: if a student has found it extremely difficult to understand and perform the activities in the classroom, 
then he should score 1 in response. If, however, this same student found no difficulty, so he should score 7 in response. In this context, the following responses were obtained: (1) relevance- 6.23 was the average of the answers, indicating that students consider the content of the discipline very relevant for their formation; (2) interest -5.62 was the average of the answers, indicating that students have a high interest in the addressed topics (even though this value is lower than the "relevance" one); (3) difficulty-4.69 was the average of the answers, indicating that students have found some difficulties during the semester; (4) usefulness - 6.31 was the average of the answers (the highest one), indicating that students consider the content of the discipline extremely useful; (5) will use in the future-5.92 was the average of the answers, indicating that a good number of students pretend to use the discipline's content in the future; (6) teaching time-3.62 was the average of the answers, indicating that students consider that the teaching time was very suitable, neither too short nor too long - that is why the average was near 4; (7) major importance- $82 \%$ of the students pointed that algorithmic-parametric modeling and rapid prototyping were the most important resources presented to them in the discipline.

\section{Final Remarks and Future Research Work}

This didactic experience focused on a teaching/learning approach that seeks to support students' design processes, through the introduction of algorithmic-parametric modeling and rapid prototyping techniques. This meant showing new creative possibilities, allowing the handling of laser cutting and 3D printing machines and, fundamentally, stimulating the exploitation of what these new means provide, with their potentialities and difficulties.

The use of $3 \mathrm{D}$ printers and laser cutting machine was, therefore, an important aspect to be considered, from the point of view of the didactic process. For the first time in their undergraduate courses, the students had the opportunity to see their projects materialized, with the precision inherent in this equipment. This meant, according to the students themselves, an improvement in the possibilities of comprehension of their ideas, in relation to conventional visualization. In this sense, the perception that the presented discipline (and the didactic experience itself) contributed to increase the students' capabilities about geometric and logic attributes was reinforced.

Although some students recognized the difficulty of assimilating the discipline's content, especially regarding the commands and the practice transmitted in the classes, the final works presented a significant improvement in their understanding and use of computational resources in design tasks. It is also important to highlight that most of the students demonstrated interest in continuing to research in this field after completing the course. In summary, the students' evaluation results showed that the discipline content is relevant, useful and interesting. However, they found some difficulty in understanding and performing the proposed activities in the classroom, which suggests some modifications for the classes dynamics in the semesters to come. It is important to highlight that they considered the teaching time suitable and that they considered algorithmic-parametric modeling and rapid prototyping techniques as the most important "topics" presented to them in the discipline. In this scenario, it is important to realize that the implementation of algorithmic-parametric logic in design processes demands a certain knowledge of mathematics/programming, because it is from this knowledge that it becomes feasible to represent both algorithmically (Grasshopper definitions) and visually, the basic shape of a design solution. Hence, in order to reduce the difficulty of students in classrooms' activities, in the next semesters, it is intended to focus, initially, on basic mathematical/logic concepts. The students, in this context, may begin earlier to 
understand how the modification of parameters and rules of generation influence the final form and how the relation between logic, mathematics and architecture occurs.

Finally, it is very important to raise awareness in those responsible for architecture courses in Brazil about the importance of increasing programming contents in our pedagogic agenda, as well as to reinforce the possibilities of its use in other subjects.

\section{References}

[1] Achten, H. 2003. "New Design Methods for Computer Aided Architectural Design Methodology Teaching." International Journal of Architectural Computing 1: 72-91.

[2] Al-Ratrout, S., and Zureikat, R. 2014. "Pedagogic
Approach in the Age of Parametric Architecture: Experimental Method for Teaching Architectural Design Studio to 3rd Year Level Students." In Proceedings of ASCAAD 2014, Jeddah, 221-6.

[3] Aguiar, R. and Gonçalves, A. 2015. "Programming for Architecture: The Students' Point of View." In Proceedings of the 33rd eCAADe Conference, Vienna, 159-68.

[4] Mitchell, W. 1990. The Logic of Architecture. Cambridge: MIT Press.

[5] Menges, A. 2006. Instrumental Geometry Architectural Design: Techniques and Technologies in Morphogenetic Design. London: Editorial Offices.

[6] Woodbury, R. 2010. Elements of Parametric Design. New York: Routledge.

[7] Tedeschi, A. 2014. Algorithms-Aided Design: Parametric Strategies Using Grasshopper. Brianza: Le Penseur.

[8] Terzidis, K. 2006. Algorithmic Architecture. New York: Routledge. 